\title{
A Novel Design Strategy for Temperature-Responsive IPN Hydrogels Based on a Copolymer of Acrylamide and N-(1,1-Dimethyl-3-0xobutyl)-Acrylamide
}

\author{
Yu Wang1,2*, Huifang Xia1, Jun Zhao', Xiuqin Cai ${ }^{3}$, Shengke Chen1, Bengxiu Li ${ }^{3,4}$ \\ ${ }^{1}$ School of Chemistry and Materials Sciences, Guizhou Education University, Guiyang, China \\ ${ }^{2}$ Anshun Engineering Research Centre of Concrete Admixture, Anshun, China \\ ${ }^{3}$ School of Chemistry and Materials Science, Weinan Normal University, Weinan, China \\ ${ }^{4}$ GuizhouTiejinahengfa New Material Technology Co. Ltd., Anshun, China \\ Email: *yu.wang@live.com
}

How to cite this paper: Wang, Y., Xia, H.F., Zhao, J., Cai, X.Q., Chen, S.K. and Li, B.X. (2018) A Novel Design Strategy for Temperature-Responsive IPN Hydrogels Based on a Copolymer of Acrylamide and N-(1,1-Dimethyl-3-Oxobutyl)-Acrylamide. Advances in Chemical Engineering and Science, 8, 255-270.

https://doi.org/10.4236/aces.2018.84018

Received: September 18, 2018

Accepted: October 16, 2018

Published: October 19, 2018

Copyright $\odot 2018$ by authors and Scientific Research Publishing Inc. This work is licensed under the Creative Commons Attribution International License (CC BY 4.0).

http://creativecommons.org/licenses/by/4.0/ (c) (i) Open Access

\begin{abstract}
A methology is described for the synthesis of novel temperature-responsive interpenetrating polymer network (IPN) hydrogels with poly(2-acrylamido2-methylpropane sulfonic acid) (PAMPS) as a tightly crosslinked 1st network, temperature-responsive poly(acrylamide-co-N-(1,1-dimethyl-3-oxobutyl)acrylamide) (P(AM-co-DAAM)) with low cost as a lossely crosslinked 2nd network. The structure and morphology of the IPN hydrogels were characterized by FTIR, TGA and SEM, and the results indicated that PAMPS network introduced $\mathrm{P}(\mathrm{AM}-\mathrm{co}-\mathrm{DAAM})$ hydrogels have large, thermally stable and interconnected porous network. The properties of the IPN hydrogels, which include: swelling capacity, equilibrium swelling/deswelling ratio, temperatureresponsive behavior, and the dwelling kinetics as specific temperature, were investigated carefully. Results showed that the obtained IPN hydrogels displayed a controllable equilibrium swelling/deswelling behavior and possessed remarkable thermosensitivity. In addition, the results also indicate that the incorporation of the hydrophobic groups DAAM has a big effect on the LCST of the IPN hydrogels. Consequently, these novel temperature-responsive IPN hydrogels with low cost and slow-releasing performance would be promising for potential applications, such as environmental catalysis, water treatment, and agriculture.
\end{abstract}

\section{Keywords}

Temperature-Responsive, Hydrogel, Interpenetrating Polymer Network, Equilibrium Swelling Ratio, Slow-Releasing Performance 


\section{Introduction}

Hydrogels are three-dimensional, hydrophilic, polymeric networks having a solid-like appearance that does not dissolve in water but can absorb large amounts of water and aqueous ingredients [1] [2] [3]. Over the past few decades, hydrogels have become one of the most extensively studied soft materials and currently continue to fascinate researchers throughout the world. In recent years, stimuli-responsive hydrogels as intelligent materials are increasingly attracting the academic and industrial interests: these hydrogels can undergo abrupt volume or phase transition in response to environmental stimuli such as temperature [4], $\mathrm{pH}$ [5], light [6], electric field [7], magnetic field [8], and oxidation-reduction [9], etc. Because of this unique feature, stimuli-responsive hydrogels have received extensive attention in the fields of controlled drug delivery [10], separation [11], tissue engineering [12], soft robtics [13], artificial muscles [14], catalysis [15], and solving environmental problems [16], etc. Among these stimuli-responsive hydrogels, temperature-responsive hydrogels are the most widely investigated.

Temperature-responsive hydrogels demonstrate a good hydrophilicity in aqueous solutions at low temperature, and separate from the solution when the temperature is raised above the lower critical solution temperature (LCST). Poly(N-isopropyl acrylamide) (PNIPAAm) hydrogel is typical temperature-responsive polymeric network, which exhibits phase separation at its relative low LCST of $32^{\circ} \mathrm{C}-34^{\circ} \mathrm{C}$ in aqueous solution [17]. At a temperature lower than the LCST, the PNIAAm hydrogel can absorb water and exist in swollen state because of the bonding interaction between the hydrophilic amide group and water molecules. Whereas, at a temperature higher than the LCST, the hydrogel undergoes an abrupt and dramatic shrinkage in volume due to the disruption of hydrogen bonds and hydrophobic interactions among the isopropyl groups of neighboring polymer chains.

From the viewpoint of applications, although the temperature-responsive hydrogels based on $\mathrm{N}$-isopropylacrylamide (NIPAM) have been investigated for many biomedical and pharmaceutical applications [18] [19] [20], the challenge impeding their potential applications such as environmental catalysis [21] [22] [23], water treatment [24] [25], and agriculture [26] is encountered. The main limitation of the conventional PNIPAAm hydrogel is fairly expensive, which may impede large-scale manufacturing of the temperature-responsive hydrogels and acceptance of water treatment and agriculture, and its phase transition is limited in a narrow range. Also, PNIPAAm hydrogels are limited by their poor mechanical properties, with the modulus and strength values [27] [28].

Based on consideration of the above limitations, studies have shown that temperature-responsive polymers on acrylamide (AM)/N-(1,1-dimethyl-3oxobutyl)-acrylamide) (DAAM), as a component of hydrogel, can effectively reduce the cost of temperature-responsive hydrogels [29] [30] and hydrogels 
consisting of two independently crosslinked polymers networks can achieve improved mechanical properties [31] [32].

In this report, novel temperature-responsive interpenetrating polymer network(IPN) hydrogels were prepared with a tightly crosslinked, highly negatively charged poly(2-acrylamide-2-methyl-propane sulfonic acid) (PAMPS) 1st network, and loosely crossliked, neutral temperature-responsive P(AM-co-DAAM) with low cost, which the LCST value of that can be controlled by varying the AM/DAAM mass ratio according to the application field, 2nd network. The structural characterizations and the thermal properties of these temperature-responsive IPN hydrogels were done by Fourier Transform Infrared Spectroscopy (FTIR), field emission scanning electron microscopy (SEM), thermogravimetric (TGA), and differential scanning calorimetric (DSC) analyses. These temperature-responsive IPN hydrogels with lowcost can have practical applications to environmental catalysis and water treatment.

\section{Materials and Methods}

\subsection{Reagents and Materials}

2-acrylamido-2-methylpropane sulfonic acid (AMPS, 97\%) was obtained from Sigma-Aldrich. Acrylamide (AM, 99.5\%, ChangjiuAgri-Scientific Co. Ltd, Nanchang, China) and N-(1,1-dimethyl-3-oxobutyl)-acrylamide (DAAM, >98\%, Liangxi Fine Chemicals Co. Ltd., Wuxi, China) were recrystallized twice from methanol and dried under vacuum prior to use. N,N-methylenebis(acrylamide) (MBA) (Sinopharm Chemical Reagent Co., Ltd., China) used as across-linking agent was recrystallized from ethanol. 2-oxoglutaric acid (Sinopharm Chemical Reagent Co., Ltd., China) was used as an initiator. The water used was doubly distilled in an all-glass apparatus, and the nitrogen gas was $99.999 \%$ in purity.

\subsection{Preparation of Temperature-Responsive IPN Hydrogels}

The temperature-responsive IPN hydrogel was prepared via a two-step strategy, as shown in Scheme 1. In the first step, the required masses of AMPS monomer, photoinitiator, crosslinker were dissolved in deionized water. Nitrogen was bubbled through the monomer/solvent mixture for $30 \mathrm{~min}$ toremove oxygen dissolved in the reaction mixture. The solution was cast on glass plates equipped with spacers, then photo-polymerized by UV lamp with full wavelength at $20^{\circ} \mathrm{C}$ for $2 \mathrm{~h}$. The hydrogel was then removed from the plates and immersed in deionized water to remove the unreacted monomers. The hydrogel was taken out and placed in fresh deionized water three times a day for 7 days before it was dried first in air and then dried in a vacuum oven. In the second step, the dried PAMPS hydrogel, of known weight, was immersed in $40 \mathrm{~mL}$ of aqueous containing desired amounts of AM, DAAM, 2-oxoglutaric acid (as photointiator) and MBA (crosslinker) for at least 3 days until the equilibrium was reached. The soft-swollen hydrogels were gently handled and kept between two glass plaques 


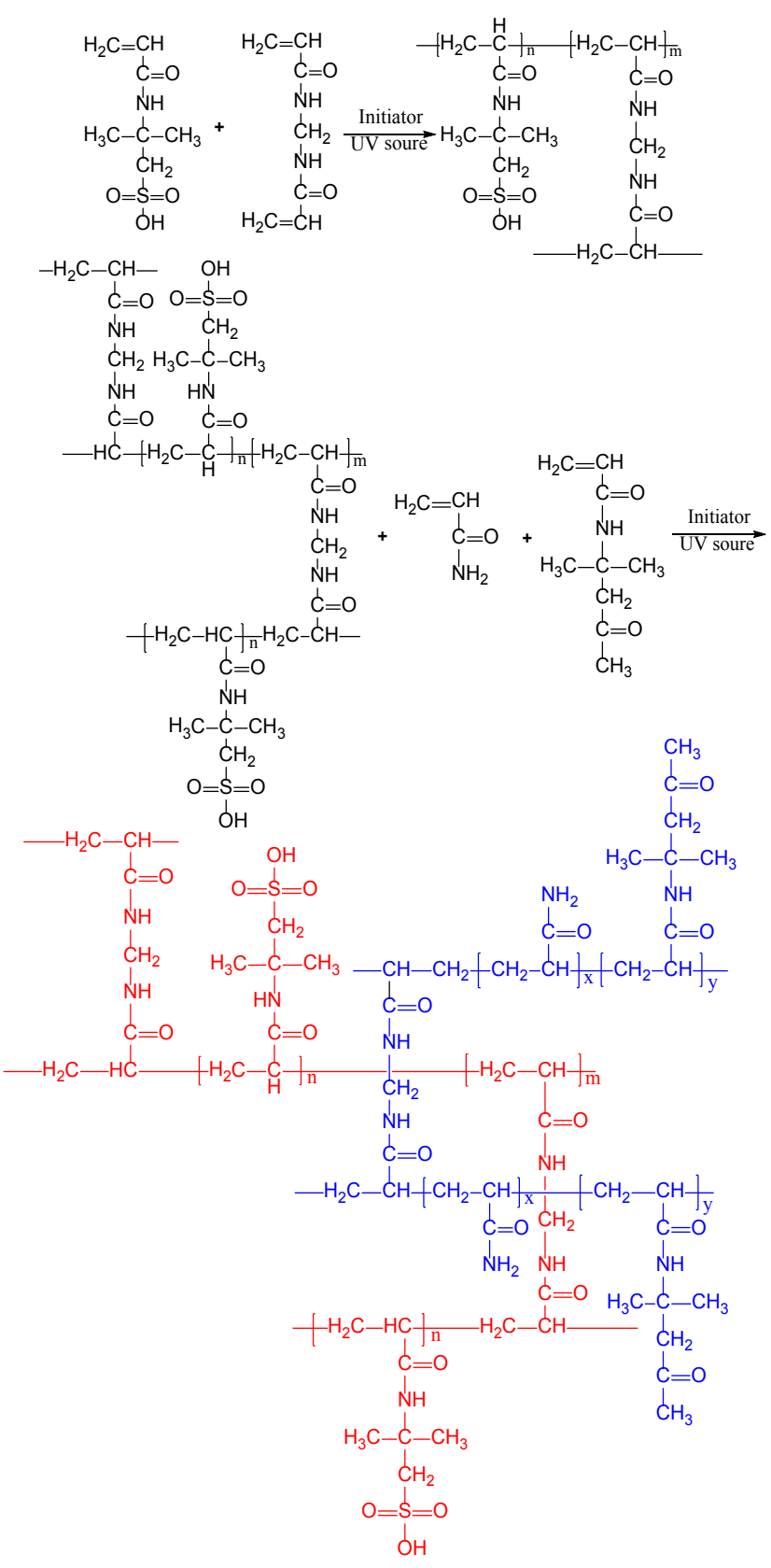

Scheme 1. The synthetic procedure of temperature-responsive IPN hydrogel.

separated by a rubber gasket spacer. By irradiation with the UV lamp for $8 \mathrm{~h}$ (the distance between the lamp and the sample chamber was about $15 \mathrm{~cm}$ ), the second network was subsequently synthesized in the presence of the first network. During the polymerization reaction, the temperature inside the chamber rose up to $40^{\circ} \mathrm{C}-50^{\circ} \mathrm{C}$ and after $1-2 \mathrm{~h}$ the hydrogel became opalescent indicating the formation of temperature-responsive poly(acrylamide-co-N-(1,1-dimethyl3-oxobutyl)-acrylamide) (P(AM-co-DAAM)) network. The as-formed IPN hydrogels, hereafter labeled as IPN1, IPN2, IPN3, IPN4 and IPN5 were dipped in distilled water for 7 days at room conditions for removing unreacted moieties. 
The water was renewed every $12 \mathrm{~h}$. After, the hydrogels were dried at room temperature. The feed compositions of the hydrogels' synthesis reaction are shown in Table 1.

\subsection{Temperature-Responsive IPN Hydrogels Characterization}

\subsubsection{Fourier Transform Infrared Spectroscopy (FTIR)}

The FTIR spectra of the dried hydrogel samples were recorded by making KBrpellets on a Nicolet MX-1E FTIR spectrophotometer (USA). The FTIR spectra were recorded in the range of $400-4000 \mathrm{~cm}^{-1}$.

\subsubsection{Thermo-Gravimetric Analysis (TGA)}

TGA was carried out with a NETZSCH STA 409 C/CD instrument under an oxygen free nitrogen atmosphere. Dry samples of $5-8 \mathrm{mg}$ weight were used. A linear temperature heating rate of $10^{\circ} \mathrm{C} \cdot \mathrm{min}^{-1}$ was maintained from $30^{\circ} \mathrm{C}$ to $900^{\circ} \mathrm{C}$. TGA weight loss curves were recorded.

\subsubsection{Scanning Electron Microscopy (SEM)}

SEM was performed on hydrogels after freeze-dried to maintain the porous structure without any collapse. The samples were plunged in liquid nitrogen, and the vitrified samples were cut with a cold knife. They were mounted on the base plate and coated with gold. The morphology was imaged on a Hitachi S-570 SEM (Tokyo, Japan) using an accelerating voltage of $20 \mathrm{kV}$.

\subsubsection{Measurement of Swelling Kinetics}

The swelling kinetics of the hydrogels was measured at $20^{\circ} \mathrm{C}$. After wiping off water or $0.9 \mathrm{wt} \% \mathrm{NaCl}$ solution on the surface with filter paper, the $S R$ of the hydrogel was recorded during the course of swelling at regular time intervals. The $S R$ was calculated by Equation (1)

$$
S R=\frac{W_{t}-W_{d}}{W_{d}}
$$

where $W_{t}$ is the weight of wet hydrogel at regular time intervals and $W_{d}$ is the weight of the dried hydrogel.

\subsubsection{Measurement of Deswelling Kinetics}

The kinetics of deswelling behavior of the hydrogels was measured at $50^{\circ} \mathrm{C}$. Before the measurement of deswelling kinetics, the hydrogels were reached swollen equilibrium in deionized water or $0.9 \mathrm{wt} \% \mathrm{NaCl}$ solution at $20^{\circ} \mathrm{C}$. The weights of the hydrogels were recorded during the course of deswelling at regular time intervals after wiping off water or $0.9 \mathrm{wt} \% \mathrm{NaCl}$ solution on the surface with filter paper. The deswelling ratio $(W R)(\%)$ is defined as follows:

$$
W R(\%)=\frac{W_{t}-W_{d}}{W_{S}}
$$

where $W_{t}$ is the mass of hydrogels at time $t, W_{d}$ is the mass of the dried hydrogels, and $W_{s}$ is themass of water in the swollenhydrogels at $20^{\circ} \mathrm{C}$. 
Table 1. The feed compositions of the hydrogels' synthesis reaction.

\begin{tabular}{cccc}
\hline \multirow{2}{*}{ Sample } & First network & \multicolumn{2}{c}{ Second Network } \\
\cline { 2 - 4 } & AMPS $(\mathrm{wt} \%)$ & (DAAM:AM) Molar ratio & $(\mathrm{DAAM}+\mathrm{AM})(\mathrm{wt} \%)^{*}$ \\
\hline IPN1 & $25 \%$ & $2: 1$ & 30 \\
IPN2 & $25 \%$ & $3: 2$ & 30 \\
IPN3 & $25 \%$ & $1: 1$ & 30 \\
IPN4 & $25 \%$ & $1: 1$ & 20 \\
IPN5 & $25 \%$ & $1: 1$ & 40 \\
\hline
\end{tabular}

${ }^{*}$ The solution concentration of AM and DAAM used during polymerization of P(AM-co-DAAM)/PAMPS in Step 2.

\subsubsection{Equilibrium $S R$ at Different Temperatures}

The equilibrium $S R$ of the hydrogel was measured after wiping off water or $0.9 \%$ $\mathrm{NaCl}$ solution on the surface with filter paper in the temperature range from $20^{\circ} \mathrm{C}$ to $60^{\circ} \mathrm{C}$, hydrogel samples were immersed into excess deionized water or $0.9 \% \mathrm{NaCl}$ solution for $24 \mathrm{~h}$ at every temperature. The $S R_{e q}$ at different temperatures were calculated as Equation (1).

\subsubsection{Differential Scanning Calorimetry (DSC)}

The DSC studies were performed on a Perkin-Elmer DSC7. The samples were heated from $8^{\circ} \mathrm{C}$ to $60^{\circ} \mathrm{C}$, with a heating rate of $2^{\circ} \mathrm{C} \cdot \mathrm{min}^{-1}$ in an inert condition.

\subsubsection{Oscillating Swelling/De-Swelling Kinetics of IPN Hydrogels}

Pre-weighted dried hydrogel samples were first immersed in deionized water at $20^{\circ} \mathrm{C}$ to reach equilibrium, whereafter the oscillatory swelling behavior was observed in deionized water at alternate temperatures of $20^{\circ} \mathrm{C}$ and $60^{\circ} \mathrm{C}$. After $30 \mathrm{~min}$ of de-swelling at $60^{\circ} \mathrm{C}$, the hydrogels were reimmersed in deionized water of $20^{\circ} \mathrm{C}$ for another $30 \mathrm{~min}$ swelling. The measurement of the $S R$ for the hydrogel was performed by repeating about steps for $330 \mathrm{~min}$.

\section{Results and Discussion}

\subsection{FTIR Spectra of the Temperature-Responsive IPN Hydrogel}

The FTIR analysis of the different hydrogel samples (Figure 1) showed the presence of peaks corresponding to the functional groups of the monomeric units used in preparing the PAMPS hydrogel and IPN hydrogel. The characteristic absorption peaks of AMPS, AM and DAAM units appear at their usual wave numbers. The peaks at 1220 and $1039 \mathrm{~cm}^{-1}$ correspond to the asymmetric and symmetric $\mathrm{S}-\mathrm{O}$ stretching of the $-\mathrm{SO}_{3} \mathrm{H}$ in the AMPS units, respectively. The peak at $1650 \mathrm{~cm}^{-1}$ is due to $\mathrm{O}=\mathrm{C}-\mathrm{N}$ of AMPS, AM and DAAM, and the band around $1705 \mathrm{~cm}^{-1}$ is assigned to the characteristic stretching vibration of $\mathrm{O}=\mathrm{C}-\mathrm{CH}_{3}$ from ketone in DAAM. These results demonstrate that both the PAMPS network and the P(AM-co-DAAM) network are present in the temperature-responsive IPN hydrogel. 


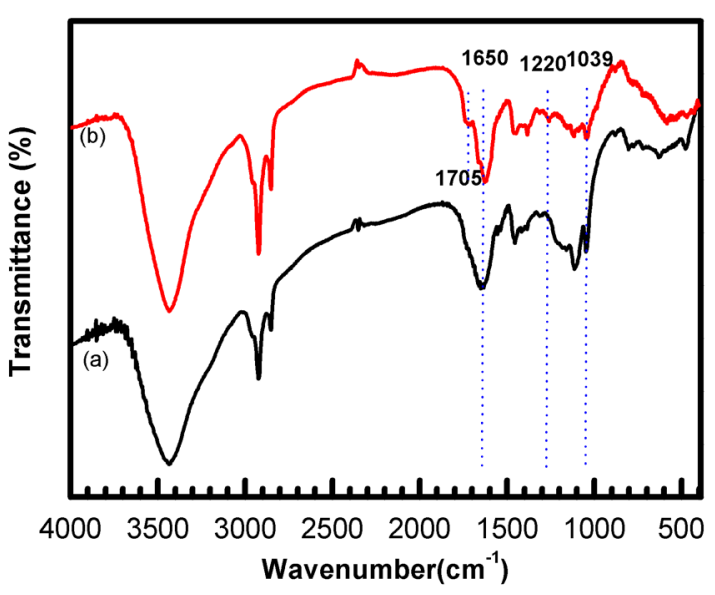

Figure 1. The FTIR spectra of PAMPS hydrogel (a), and IPN1 hydrogel (b).

\subsection{Thermogravimetric Analysis of IPN1 Hydrogel}

The thermal decomposition and weight loss profiles of PAMPS and IPN1 hydrogels were estimated from TGA thermogram as a function of temperature, as shown in Figure 2. PAMPS hydrogel presents three main thermal degradation events in the temperature range of $30^{\circ} \mathrm{C}-200^{\circ} \mathrm{C}, 200^{\circ} \mathrm{C}-400^{\circ} \mathrm{C}$, and $400^{\circ} \mathrm{C}$ $800^{\circ} \mathrm{C}$. The first event is assigned to the evaporation of residual water, where the weight loss of $10.0 \%$ took place. In the second stage almost $57.5 \%$ mass loss occurred. The third stage started from $400^{\circ} \mathrm{C}$, and $11.0 \%$ decomposition was observed at $800^{\circ} \mathrm{C}$. The latter two events were attributed to a sophisticated process by which breakage of crosslinking bridges, scission of the long chain backbone, and decomposition of imides and amide were dominant [33] [34]. Compared to PAMPS hydrogel, IPN1 hydrogel presented a higher residual mass and the onset degradation temperature emerged at higher temperature $\left(220^{\circ} \mathrm{C}\right)$, which suggested the formation thermally stable network that could be attributed to the formation of inter and intra molecular hydrogen bonds among the PAMPS and the $\mathrm{P}(\mathrm{AM}-\mathrm{co}-\mathrm{DAAM})$ chains.

\subsection{SEM Micrographs of IPN1 Hydrogel}

The interior morphology of IPN1 hydrogel is shown in Figure 3. The SEM images indicate that the interpenetrating polymer network hydrogel has been synthesized. By SEM observation, the IPN1 hydrogel appeared to have more compact porous structures with an average pore size of about $25 \mu \mathrm{m}$, due to the presence of the $\mathrm{P}(\mathrm{AM}-\mathrm{co}-\mathrm{DAAM})$ network, which increased the relative crosslink density of hydrogel structure. Moreover, due to the presence of the $\mathrm{P}(\mathrm{AM}$-co-DAAM) network, the IPN1 hydrogel shows a more porous network structure in character, which could increase the deswelling rate of the hydrogel when the temperature is about the hydrogel's LCST.

\subsection{Swelling Kinetics of the IPN Hydrogels}

In order to determine the effects of the contents of DAAM and AM on the network 


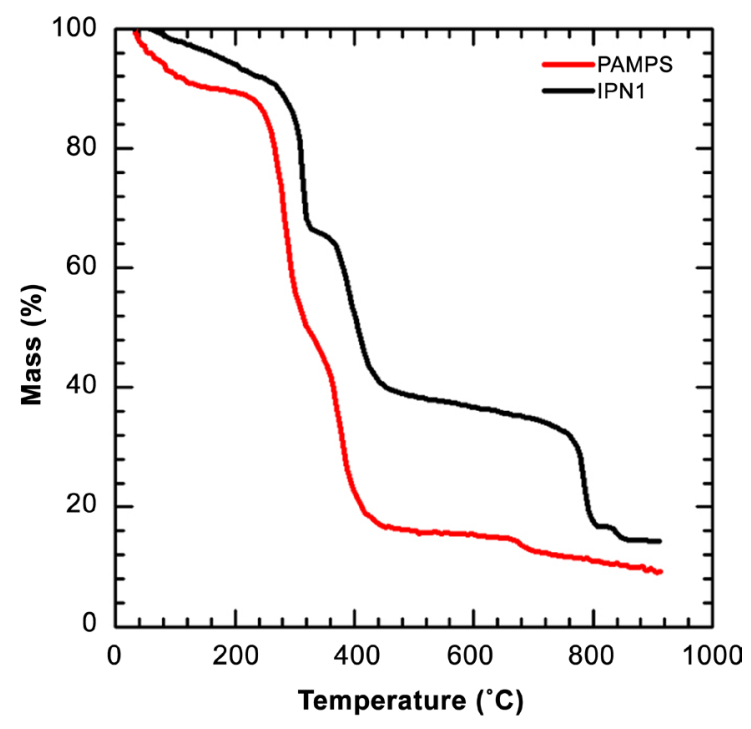

Figure 2. TGA thermograms of PAMPS and IPN1 hydrogels.
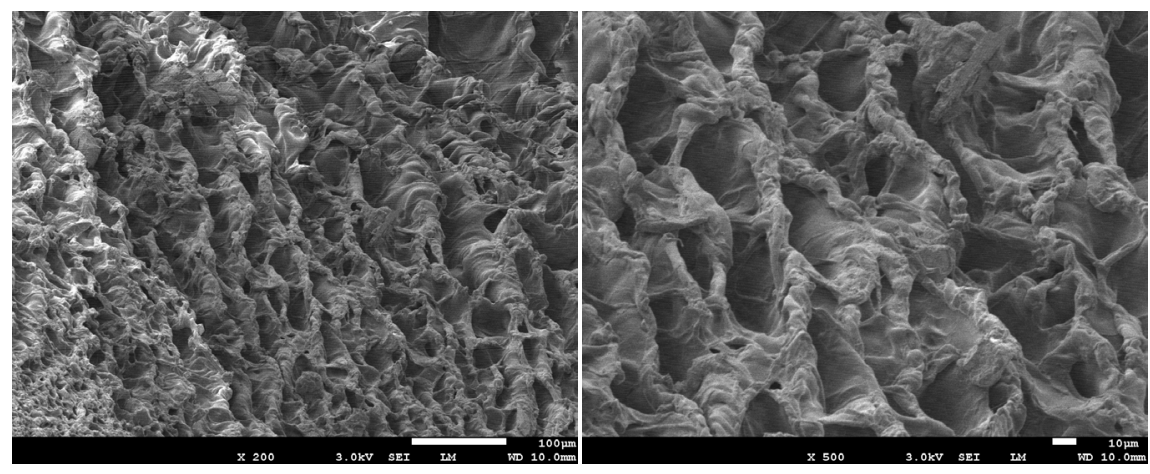

Figure 3. SEM images of the IPN1 hydroge.

density of the IPN hydrogels which were prepared by the varying the DAAM/AM molar ratio and the concentration of DAAM and AM, the swelling studies of the IPN hydrogels were carried out at $20^{\circ} \mathrm{C}$ in deionized water and 0.9 wt\% $\mathrm{NaCl}$ solution. As shown in Figure 4, the swelling properties of the IPN hydrogels with different compositions differed greatly in deionized water and 0.9 wt\% $\mathrm{NaCl}$ solution. It can be observed: 1) the $S R$ of the IPN hydrogels increased steeply within $480 \mathrm{~min}$, and then reached a plateau. 2) the $S R$ in $0.9 \mathrm{wt} \% \mathrm{NaCl}$ solution is lower than indeionized water, for example, the $S R$ of IPN3 (molar rate DAAM $/ \mathrm{AM}=1 / 1$ ) in deionized water is about $13.6 \mathrm{~g} / \mathrm{g}$ within $480 \mathrm{~min}$, while the $S R$ of it in $0.9 \mathrm{wt} \% \mathrm{NaCl}$ solution is about $3.5 \mathrm{~g} / \mathrm{g}$ within $480 \mathrm{~min} .3$ ) the $S R$ of the hydrogels decreased with an increase of DAAM content in the IPN hydrogels. For instance, the $S R$ of the IPN hydrogels in deionized water decreased from $13.6 \mathrm{~g} / \mathrm{g}$ to $9.5 \mathrm{~g} / \mathrm{g}$ as DAAM/AMmolar ratioincreased from $1 / 1$ to 2/1 within $480 \mathrm{~min}$. The phenomenon can be attributed to the enhancement in hydrophobicity of the IPN hydrogel, which renders it more and more difficult for water molecules to penetrate into the hydrogel, hence decrease the swelling ratio. Ionic strength can play important role in the swelling behaviour. Hydrogels 


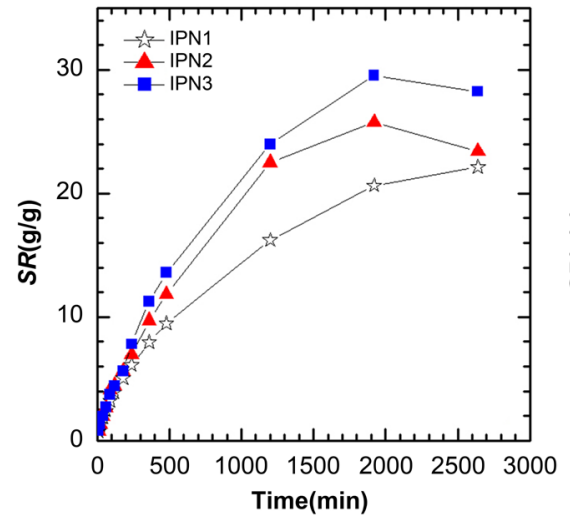

(a)

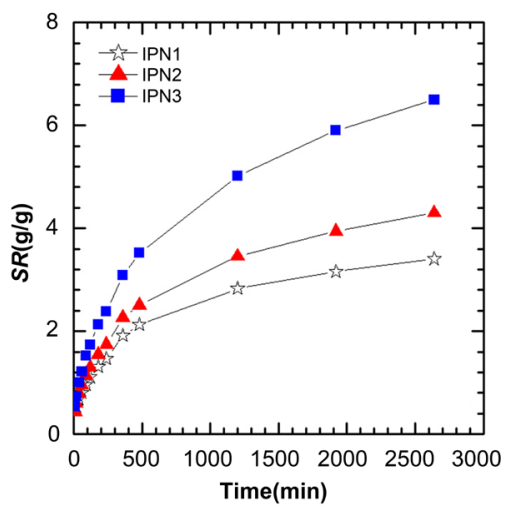

(c)

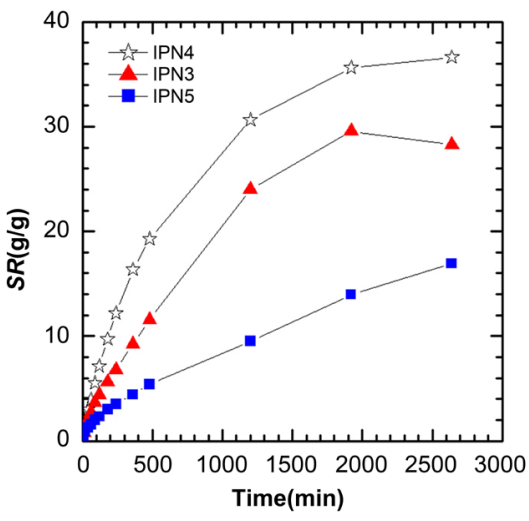

(b)

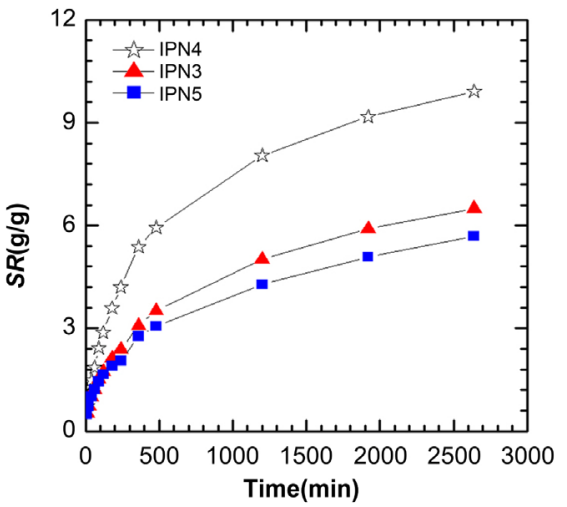

(d)

Figure 4. Swelling kinetics of IPN hydrogels in deionized water (a), (b) and in 0.9 wt\% $\mathrm{NaCl}$ solution (c), (d) at $20^{\circ} \mathrm{C}$.

do not swell appreciably in the presence of electrolytes due to the increase of movable counterions of asolution, which lead to a decrease in the osmotic pressure within the hydrogel, causing the hydrogel to shrink [35].

\subsection{Deswelling Kinetics of the IPN Hydrogels}

The investigation of deswelling kinetics is important for the temperature-responsive IPN hydrogels in measuring their water retention and deswelling rate. Figure 5 shows the deswelling kinetics of the temperature-responsive IPN hydrogels from the equilibrium swelling state at $20^{\circ} \mathrm{C}$ water bath to $50^{\circ} \mathrm{C}$ water bath. As expected, all the swollen IPN hydrogels tended to shrink and lose water after immersing in deionized water or $0.9 \mathrm{wt} \% \mathrm{NaCl}$ solution at higher temperature due the disruption of hydrophilic/hydrophobic balance in IPN hydrogels. The water retention decreased rapidly with the increase of deswelling time before reaching a constant value within $600 \mathrm{~min}$. The data illustrate that the deswelling rate ofthe IPN hydrogel samples is obviously dependent on the DAAM content. For example, the $W R$ of the IPN hydrogels decreased from $76.2 \%$ (IPN1) to $44.7 \%$ (IPN3) as DAAM/AM molar ratioincreased from $1 / 1$ to $2 / 1$ in deionized water, while the $W R$ of the hydrogels increased from $49.9 \%$ (IPN1) to $64.2 \%$ (IPN3) in $0.9 \mathrm{wt} \%$ $\mathrm{NaCl}$ solution within $600 \mathrm{~min}$. 


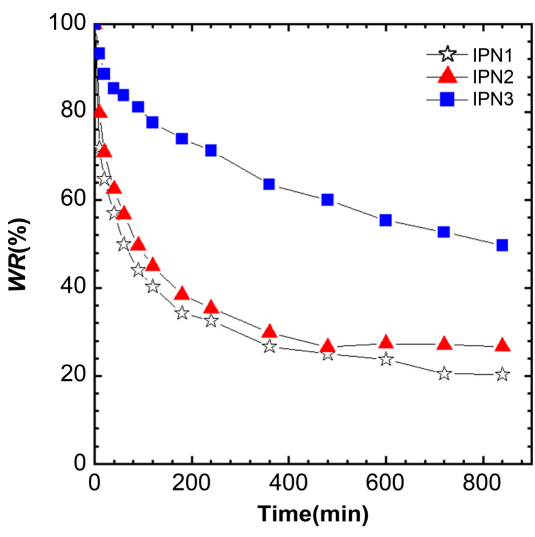

(a)

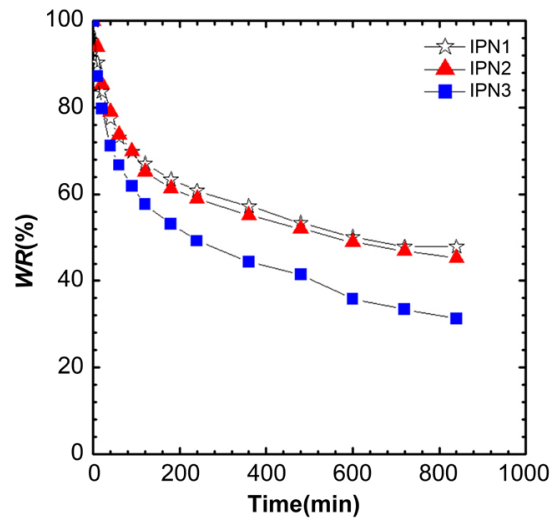

(c)

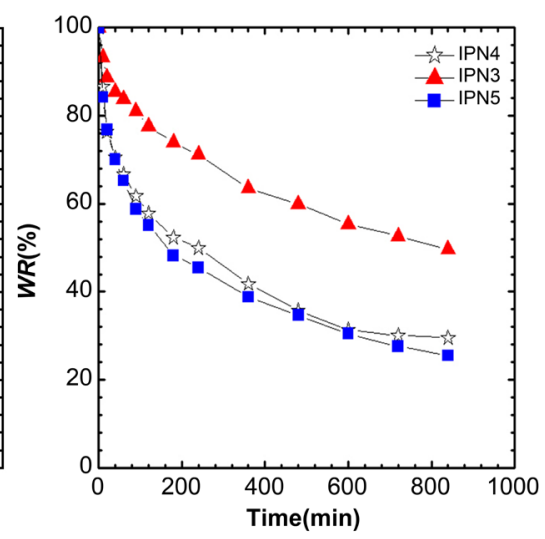

(b)

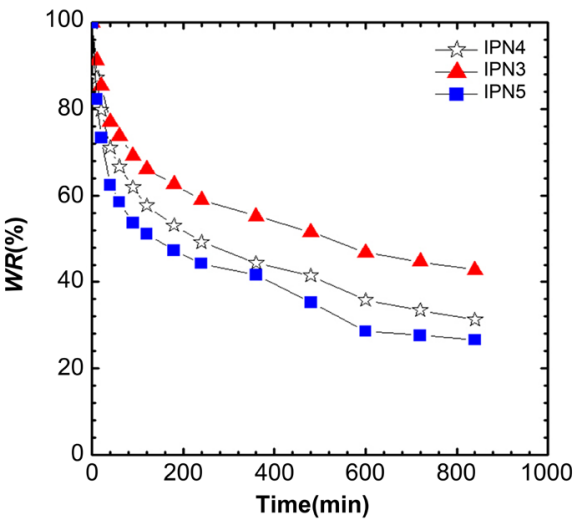

(d)

Figure 5. Deswelling kinetics of IPN hydrogels in deionized water (a), (b) and in 0.9 wt\% $\mathrm{NaCl}$ solution (c), (d) at $50^{\circ} \mathrm{C}$.

\subsection{Temperature Dependence of the IPN Hydrogels}

The equilibrium swelling ratio $(E S R)$ is one of the most important parameters for evaluating temperature-responsive hydrogels because it illustrates their LCST behavior. The effect of temperature on the ESR of the temperature-responsive IPN hydrogels in deionized water and $0.9 \mathrm{wt} \% \mathrm{NaCl}$ solution at various temperature from $20^{\circ} \mathrm{C}$ to $60^{\circ} \mathrm{C}$ are shown in Figure 6. The results show that the ESR of the IPN hydrogels decrease as the temperature increases and have a broadening hydrogel transition in the range of the temperature from $25^{\circ} \mathrm{C}$ to $60^{\circ} \mathrm{C}$. In addition, the change of the temperature-responsive IPN hydrogels from equilibrium swelling state to another is not instantaneous that the process is not in accordance with PNIPAM based hydrogels [36], indicating that the temperature-responsive IPN hydrogels Based on a copolymer of acrylamide and $\mathrm{N}$-(1,1-dimethyl-3-oxobutyl)-acrylamide were suitable for slow-releasing applications. The temperature-response of IPN hydrogels is attributed to the alteration of hydrophilicity of the network because the thermosensive P(AM-co-DAAM) is incorporated into the first hydrogel network. At temperature increase, a part of hydrogen bonds will destroyed, and the hydrophobic interactions among the hydrophobic groups in the second $\mathrm{P}(\mathrm{AM}-\mathrm{co}-\mathrm{DAAM})$ network become dominant 


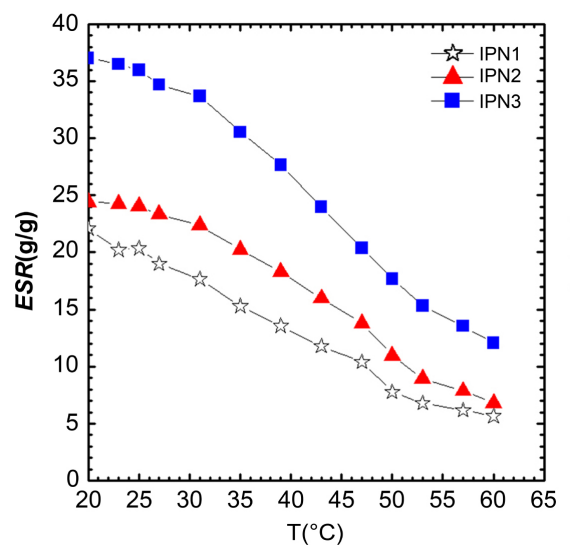

(a)

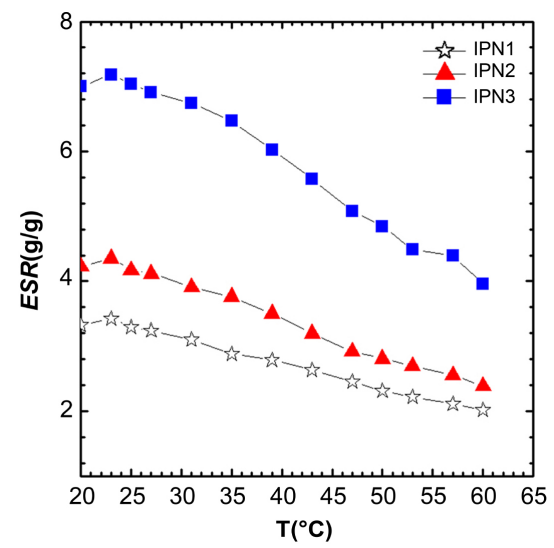

(c)

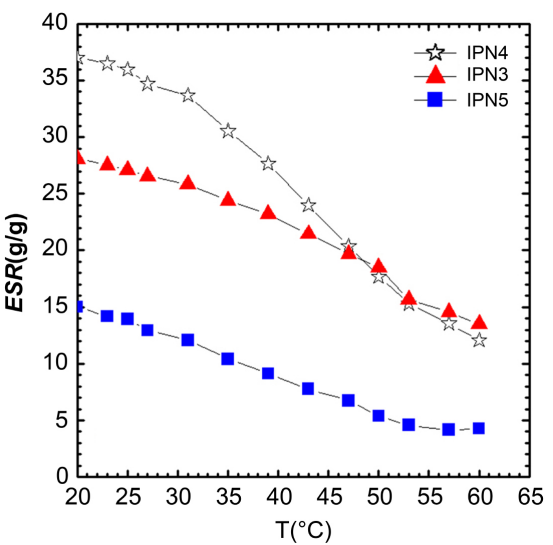

(b)

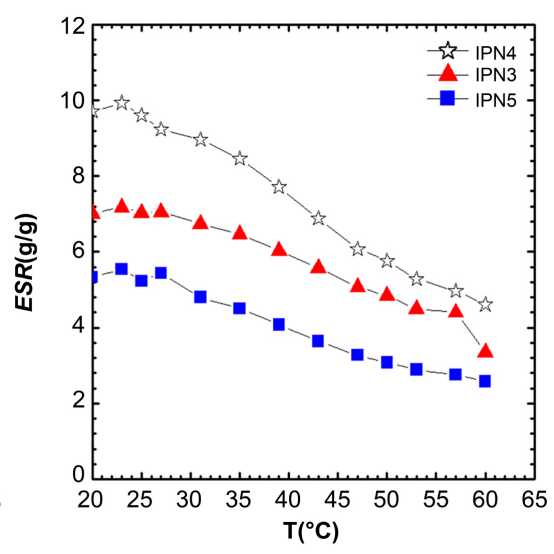

(d)

Figure 6. ESR of IPN hydrogels in deionized water (a), (b) and in $0.9 \% \mathrm{NaCl}$ solution (c), (d) over the temperature range from $20^{\circ} \mathrm{C}$ to $60^{\circ} \mathrm{C}$.

and consequently the IPN hydrogels become much less hydrophilic. With the increasing DAAM/AM molar ratio or the contents of DAAM and AM in the INP hydrogels, the hydrogels became more hydrophobic and the swelling ratio of the hydrogels decreased more sharply.

\subsection{Thermal Behavior of the IPN Hydrogels}

The thermal behavior of the IPN hydrogels was investigated using DSC with the LCST reported as the peak temperature [37] [38]. At the LCST, the water in hydrogels will be separated from the network, leading to a smaller heat capacity. As shown in Figure 7, the phase transition of the IPN hydrogels is gradually strengthened as DAAM/AM molar ratio or the concentration of DAAM and AM increases. For instances, the LCST of IPN hydrogels decreased from $39^{\circ} \mathrm{C}$ (IPN3) to $18^{\circ} \mathrm{C}$ (IPN1), as DAAM/AM molar ratio increased from $1 / 1$ to $2 / 1$. Note that the LCST of IPN4 hydrogel is blurry because of the extremely weak phase transition caused by decreasing the contents of thermosensive P(AM-co-DAAM). The results indicate that the incorporation of the hydrophobic groups DAAM has a big effect on the LCST of the IPN hydrogels, as discussed earlier. 

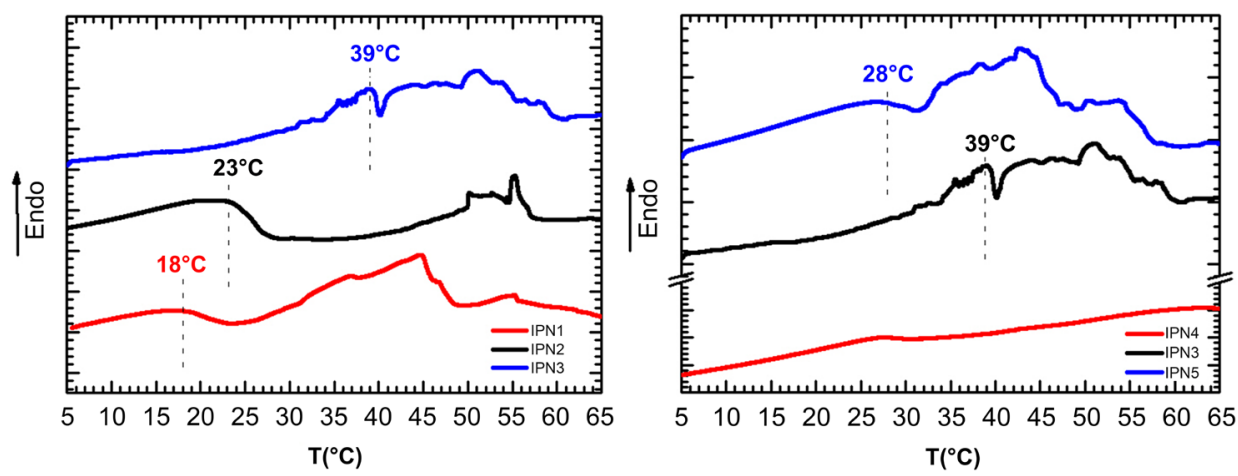

Figure 7. DSC curves of IPN hydrogels.

\subsection{Oscillatory Swelling/Deswelling Kinetics of the IPN Hydrogels}

From the point of applications, the oscillating swelling-deswelling properties over a shorter time intervals with the small temperature cycles of the hydrogels are important, which would be stable for potential applicants. So it is necessary to investigate the oscillating swelling-deswelling kinetics in response to the temperature changes. Figure 8 shows the effect of oscillatory cycling on the thermosensitivity of the synthesized IPN hydrogels at $20^{\circ} \mathrm{C}$ and $60^{\circ} \mathrm{C}$. It can be found that the $S R$ of the hydrogels decreased slightly with increasing number of cycles due to their relative slow swelling rate comparing with their shrinking rate. The slower and smaller magnitude of oscollating responses from the novel temperature-responsive IPN hydrogels may be advantageous for practical applications in many fields such as environmental catalysis, water treatment, and agriculture.

\subsection{Effect of Temperature on the Appearance of IPN Hydrogel}

The effect of temperance on the appearance of IPN hydrogel is shown in Figure 9. The results show that the change of appearance of IPN hydrogel was observed as the water temperature was switched from $20^{\circ} \mathrm{C}$ to $60^{\circ} \mathrm{C}$. When the water temperature is higher than $23^{\circ} \mathrm{C}$, the appearance of IPNhydrogel was changed from transparent to opaque because a collapsed phase transition of $\mathrm{P}(\mathrm{AM}-\mathrm{Co}-\mathrm{DAAM})$ component was occurred under water above LCST of P(AM-co-DAAM) [29]. The IPN hydrogel would be shrunk as the temperature increased. This result conforms to the above-mentioned results for the temperature effect on ESR of the IPN hydrogels.

\section{Conclusion}

A series of novel temperature-responsive IPN hydrogels based on a copolymer of acrylamide and $\mathrm{N}$-(1,1-dimethyl-3-oxobutyl)-acrylamide were successfully synthesized by a two-step method. Some conclusions can be drawn as follows. The temperature-response of the synthesized IPN hydrogels can be successfully endowed by immersing of P(AM-co-DAAM) solution into the first PAMPS network and the thermosensitivities of the IPN hydrogels are more obvious as the 

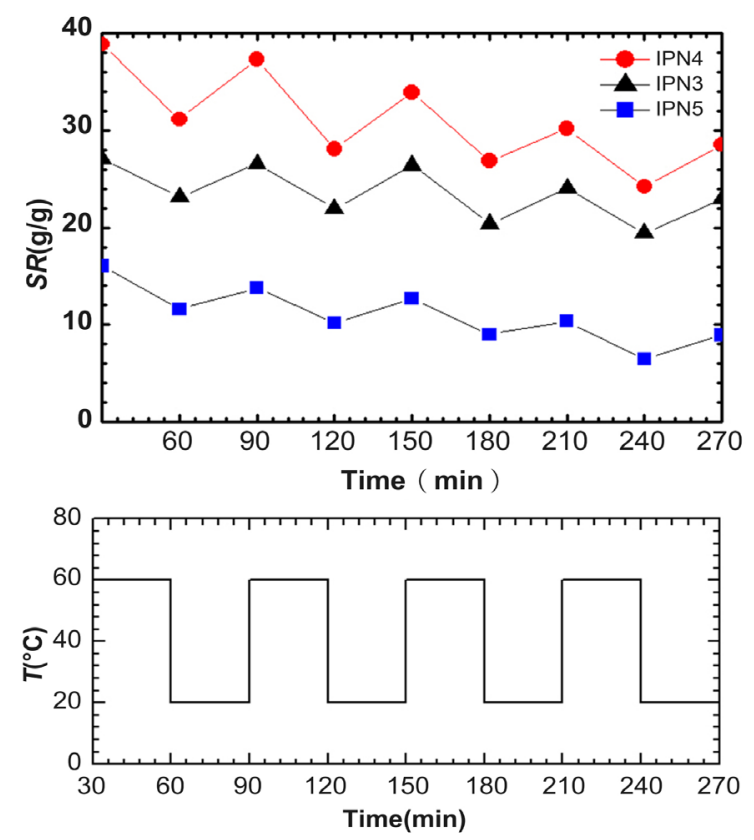

Figure 8. Oscillatory swelling/de-swelling kinetics of IPN hydrogels over 30 min temperature cycles in deionized wate rbetween $20^{\circ} \mathrm{C}$ and $60^{\circ} \mathrm{C}$.
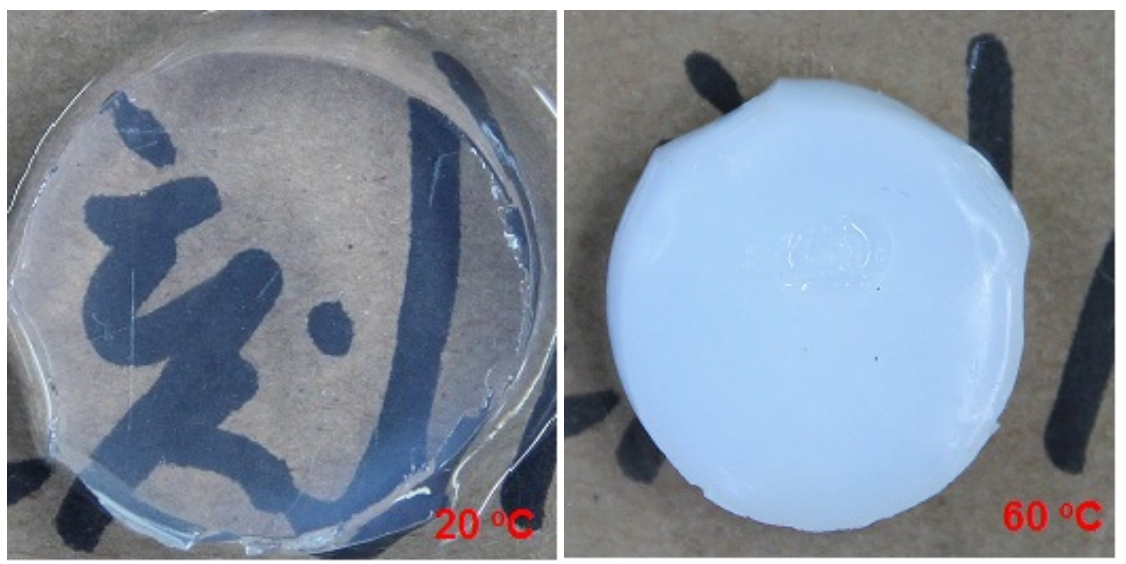

Figure 9. Photographs of swollen the IPN hydrogel in water at $20^{\circ} \mathrm{C}$ and $60^{\circ} \mathrm{C}$, respectively.

DAAM/AM molar ratio or the content of P(AM-co-DAAM) moiety in the IPN hydrogels increased. The ESR of the IPN hydrogels decreases with increasing the DAAM/AM mass ratio or the content of $\mathrm{P}(\mathrm{AM}-\mathrm{CO}-\mathrm{DAAM})$ moiety in the IPN hydrogels and the ESR in $0.9 \mathrm{wt} \% \mathrm{NaCl}$ solution is lower than in deionized water. The physical properties of temperature-responsive IPN hydrogels, such as equilibrium swelling/deswellingratio, water retention, reversible response, and temperature dependence behaviors, could be effectively controlled by the internal chemical composition and external temperature. These novel temperature-responsive IPN hydrogels with low lost, thermally stable network, tunable swelling/deswelling characteristics, and distinct thermosensitivity are promising candidates for applications in environmental catalysis, water treatment and 
agriculture.

\section{Acknowledgements}

The authors gratefully acknowledge the financial supports from National Natural Science Foundation of China (No. 21464005, 201703157), Natural Science Foundation of Guizhou Province (No. J20181122), Youth Science and Technology Talent Development Project of the Education Department of Guizhou Province, as well as the Key Disciplines Construction Foundation of Applied Chemistry of Guizhou Province (No. 2012442).

\section{Conflicts of Interest}

The authors declare no conflicts of interest regarding the publication of this paper.

\section{References}

[1] BeraM R., Dey, A. and Chalrabary, D. (2015) Synthesis, Characterizationand Drug Release Study of Acrylamide-Co-Itaconic Acid Based Smart Hydrogel. Polymer Engineering \& Science, 55, 113-122. https://doi.org/10.1002/pen.23874

[2] Drury, J.L. and Mooney, D.J. (2003) Hydrogels for Tissue Engineering: Scaffold Design Variables and Applications. Biomaterials, 24, 4337-4351. https://doi.org/10.1016/S0142-9612(03)00340-5

[3] Menezes, R., Camilo, A. and Felisberti, M.I. (2017) Thermoresponsive Hydrogels Based on Sucrose 1- $O$-Methacrylate and $N$-Isopropylacrylamide: Synthesis, Properties, and a Applications. Journal of Applied Polymer Science, 134, 45495-45509. https://doi.org/10.1002/app.45495

[4] Zhao, Z.X., Li, Z., Xia, Q.B., Xi, H.X. and Lin, Y.S. (2008) Fast Synthesis of Temperature-Sensitive PNIPAAm Hydrogels by Microwave Irradiation. European Polymer Journal, 44, 1217-1244. https://doi.org/10.1016/j.eurpolymj.2008.01.014

[5] Strehin, I., Nahas, Z., Arora, K., Nguyen, T. and Elisseeffv, J. (2010) A Versatile pH Sensitive Chondroitin Sulfate-PEG Tissue Adhesive and Hydrogel. Biomaterials, 31, 2788-2797. https://doi.org/10.1016/j.biomaterials.2009.12.033

[6] Juodkazis, S., Mukai, N., Wakaki, R., Yamaguchi, A., Matsuo, S. and Misawa, H. (2000) Reversible Phase Transitions in Polymer Gelsinduced by Radiation Forces. Nature, 408, 178-181. https://doi.org/10.1038/35041522

[7] Bajpai, A.K., Shukla, K.S., Bhanu, S. and Kankane S. (2008) Responsive Polymers in Controlled Drug Delivery. Progress in Polymer Science, 33, 1088-1118.

https://doi.org/10.1016/j.progpolymsci.2008.07.005

[8] Meenach, S.A., Hilt, J.Z. and Anderson, K.W. (2010) Poly(Ethylene Glycol)-Based Magnetic Hydrogel Nanocompositesfor Hyperthermia Cancer Therapy. Acta Biomaterials, 6, 1039-1046. https://doi.org/10.1016/j.actbio.2009.10.017

[9] Wegner, S.V., Schenk, F.C., Witzel, S., Bialas, F. and Spatz, J.P. (2016) Cobalt Cross-Linked Redox-Responsive PEG Hydrogels: From Viscoelastic Liquids to Elastic Solids. Macromolecules, 49, 4229-4235. https://doi.org/10.1021/acs.macromol.6b00574

[10] Stuart, M., Huck, W., Genzer, J., Muller, M., Ober, C., Stamm, M., Sukhorukov, G., Szleifer, I., Tsukruk, V.V., Urban, M., Winnik, F., Zauscher, S., Luzinov, I. and 
Minko, S. (2010) Emerging Applications of Stimuli-Responsive Polymer Materials. Nature Materials, 9, 101-113. https://doi.org/10.1038/nmat2614

[11] Feil, H., Bae, Y.H., Feijen, J. and Kim, S.W. (1991) Molecular Separation by Thermosensitive Hydrogel Membranes. Journal of Membrane Science, 64, 283-294. https://doi.org/10.1016/0376-7388(91)80099-R

[12] Lee, K.Y. and Mooney, D.J. (2001) Hydrogels for Tissue Engineering. Chemical Reviews, 101, 1869-1879. https://doi.org/10.1021/cr000108x

[13] Zheng, W.J., An, N., Yang, J.H., Zhou, J. and Chen, Y.M. (2015) Tough Al-Alginate/Poly( $N$-Isopropylacrylamide) Hydrogel with Tunable LCST for Soft Robotics. ACS Applied Materials \& Interfaces, 7, 1758-1764. https://doi.org/10.1021/am507339r

[14] Takashima, Y., Hatanaka, S., Otsubo, M., Nakahata, M., Kakuta, T., Hashidzume, A., Yamaguchi, H. and Harada, A. (2013) Expansion-Contraction of Photoresponsive Artificial Muscle Regulated by Host-Guest Interactions. Nature Communications, 3, 1270-1278. https://doi.org/10.1038/ncomms2280

[15] Hapiot, F., Menuel, S. and Monflier, E. (2013) Thermoresponsive Hydrogels in Catalysis. ACS Catalysis, 3, 1006-1010. https://doi.org/10.1021/cs400118c

[16] Xiao, L., Isner, A.B., Hilt, J.Z. and Bhattacharyya, D. (2013) Temperature Responsive Hydrogel with Reactive Nanoparticles. Journal of Applied Polymer Science, 128, 1804-1814.

[17] Ricka, J. and Tanaka, T. (1984) Swelling of Ionic Gels: Quantitative Performance of the Donnantheory. Macromolecules, 17, 2916-2921.

https://doi.org/10.1021/ma00142a081

[18] Ma, C., Shi, Y., Pena, D.A., Peng, L. and Yu, G. (2015) Thermally Responsive Hydrogel Blends: A General Drug Carrier Model for Controlled Drug Release. Angewandte Chemie International Edition, 54, 7484-7488.

[19] Mastiholimath, V.S., Dandragi, P.M., Jain, S.S., Gadad, A.P. and Kulkami, A.R. (2007) Time and pH Dependent Colon Specific, Pulsatile Delivery of Theophyllinefor Nocturnal Asthma. International Journal of Pharmaceutics, 328, 49-56. https://doi.org/10.1016/j.ijpharm.2006.07.045

[20] Ward, M.A. and Georgiou, T.K. (2011) Thermoresponsive Polymers for Biomedical Applications. Polymers, 3, 1215-1242. https://doi.org/10.3390/polym3031215

[21] Shi, S., Zhang, L., Wang, T., Wang, Q.M., Gao, Y. and Wang, N. (2013) Poly(NIsopropylacrylamide)-Au Hybrid Microgels: Synthesis, Characterization, Thermally Tunableoptical and Catalytic Properties. Soft Matter, 9, 10966-10970. https://doi.org/10.1039/c3sm52303a

[22] Han, Y., Yuan, X., Zhu, M., Li, S., Whitcombe, M.J. and Piletsky, S.A. (2014) A Catalytic and Shape-Memory Polymer Reactor. Advanced Functional Materials, 24, 4966-5001. https://doi.org/10.1002/adfm.201400768

[23] Parasuraman, D. and Serpe, M.J. (2011) Poly (N-Isopropylacrylamide) Microgelsfororganicdye Removal from Water. ACS Applied Materials \& Interfaces, 3, 4966-5001.

[24] Cai, Y.F. and Hu, X. (2016) A Critical Review on Draw Solutes Development for Forward Osmosis. Desalination, 391, 16-29. https://doi.org/10.1016/j.desal.2016.03.021

[25] Zhao, Q.P., Chen, N.P., Zhao, D.L. and Lu, X.M. (2013) Thermoresponsive Magnetic Nanoparticles for Seawater Desalination. ACS Applied Materials \& Interfaces, 5, 11453-11461. https://doi.org/10.1021/am403719s 
[26] Guilherme, M.R., Aouada, F.A., Fajardo, A.R., Martins, A.F., Paulino, A.T., Davi, M.F., Rubira, A.F. and Muniz, EC. (2015) Superabsorbent Hydrogels Based on Polysaccharides for Application in Agriculture as Soil Conditioner and Nutrient Carrier: A Review. European Polymer Journal, 72, 365-385. https://doi.org/10.1016/j.eurpolymj.2015.04.017

[27] Means, A.K., Ehrhardt, D.A., Whitney, L.V. and Grunlan, M.A. (2017) Thermoresponsive Double Network Hydrogels with Exceptional Compressive Mechanical Properties. Macromolecular Rapid Communications, 38, 1700351-1700356. https://doi.org/10.1002/marc.201700351

[28] Zhang, H.J., Sun, L.F., Yang, B., Zhang, Y.A. and Zhu, S.J. (2016) Novel Ginkgolide B Derivative Attenuated the Function and Expression of P-Glycoprotein at the Blood-Brain Barrier, Presenting Brain-Targeting Ability. RSC Advances, 6, 31101-31106. https://doi.org/10.1039/C5RA25248B

[29] Wang, Y., Feng, Y.J., Wang, B.Q. and Lu, Z.Y. (2010) A Novel Thermoviscosifying Water-Soluble Polymer: Synthesis and Aqueous Solution Properties. Journal of Applied Polymer Science, 116, 3516-3524.

[30] Liu, L.X., Wang, Y, Lu, Z.Y., Chen, Q.S. and Feng, Y.J. (2012) Effect of Inorganic Salts on Viscosifying Behavior of a Thermoassociative Water-Soluble Terpolymer Based on 2-Acrylamido-Methylpropane Sulfonic Acid. Journal of Applied Polymer Science, 125, 4041-4048. https://doi.org/10.1002/app.36745

[31] Chen, Q., Zhu, L., Zhao, C., Wang, Q.M. and Zheng, J. (2013) Highly Efficient Electrocatalytic Hydrogen Production by MoSx Grown on Graphene. Advanced Materials, 25, 755-755. https://doi.org/10.1002/adma.201370032

[32] Naficy, S., Razal, J.M., Whitten, P.G., Wallace, G.G. and Spinks, G.M. (2012) A pH-Sensitive, Strong Double-Network Hydrogel: Poly(Ethylene Glycol) Methyl Ethermethacrylates-Poly(Acrylic Acid). Journal of Polymer Science Part B: Polymer Physics, 50, 423-430. https://doi.org/10.1002/polb.23016

[33] Qi, X.L., Wei, W., Li, J.J., Zou, G.C., Pan, X.H., Su, T., Zhang, J.F. and Dong, W. (2017) Salecan-Based pH-Sensitive Hydrogels for Insulin Delivery. Molecular Pharmaceutics, 14, 431-440. https://doi.org/10.1021/acs.molpharmaceut.6b00875

[34] Pooley, S.A., Rivas, B.L., Carcamo, A.L. and Pizarro, G. (2009) Hydrogels from 2-(Dimethylamino)Ethylacrylate with 2-Acrylamido-2-Methyl-1-Propanesulfonic Acid: Synthesis, Characterization, and Water-Sorption Properties. Polymer Bulletin, 62, 469-485. https://doi.org/10.1007/s00289-008-0026-7

[35] Mohan, Y., Murthy, P.S., Sudhakar, H., Naidu, B., Raju, K. and Raju, M. (2006) Swelling and Diffusion Properties of Poly(Acrylamide-Co-Maleic Acid) Hydrogels: A Study with Different Crosslinking Agents. International Journal of Polymeric Materials, 55, 867-892. https://doi.org/10.1080/00914030500522539

[36] Zhang, J.T. and Jandt, K.D. (2008) A Novel Approach to Prepare Porous Poly(N-Isopropylacrylamide) Hydrogel with Superfast Shrinking Kinetics. Macromolecular Rapid Communications, 29, 593-597.

https://doi.org/10.1002/marc.200700778

[37] Tang, Y.C., Ding, Y.W. and Zhang, G.Z. (2008) Role of Methyl in the Phase Transition of Poly(N-Isopropylmethacrylamide). The Journal of Physical Chemistry B, 112, 8447-8451. https://doi.org/10.1021/jp711581h

[38] Li, Z.Q., Shen, J.F., Ma, H.W., Lu, X., Shi, M., Li, N. and Ye, M.X. (2013) Preparation and Characterization of $\mathrm{pH}$ - and Temperature-Responsive Nanocomposite Double Network Hydrogels. Materials Science and Engineering. C, 33, 1951-1957. https://doi.org/10.1016/j.msec.2013.01.004 